\title{
A Comparative Study of Dust Cleaning Methods for the Solar PV Panels
}

\author{
Mallikarjun G. Hudedmani*, Gita Joshi, Umayal R M, Ashwini Revankar \\ Department of Electrical and Electronics Engineering, KLE Institute of Technology, \\ Opposite to Airport, Gokul, Hubballi, Karnataka, India
}

\section{dai: https://doi.org/10.21467/ajgr.1.1.24-29 \\ * Corresponding Author email: \\ mallikarjunh@yahoo.com}

\author{
Article History \\ Received: 01 February 2017 \\ Revised: 13 February 2017 \\ Accepted: 16 February 2017 \\ Published: 18 February 2017

\section{Student(s) \\ - Gita Joshi \\ - Umayal R M \\ - Ashwini Revankar} \\ Academic Year: 2016-17, Even Semester \\ Course Level: Bachelor Degree \\ Course Name: BE (Electrical \& Electronics \\ Engineering) \\ Course year: $4^{\text {th }}$ year $/$ VIII ${ }^{\text {th }}$ Semester \\ Mentor(s) \\ Mallikarjun G. Hudedmani
}

\begin{abstract}
The present energy consumption scenario is clearly showing the faster depletion of fossil fuel reserves. On the other hand, energy usage index indicates the state of growth of a country. The use of renewable energy sources like solar PV, wind and such alternatives has increased in the recent years. Considerable loss in conversion efficiency of solar PV system has been noticed due to nonavailability of proper insolation and accumulation of dust particles on the panels or shading. A comparative study of various cleaning methods of solar panels has been done in this article with emphasis on innovative idea of separation of dust by electrostatic precipitator (ESP). Electrostatic precipitators uses static electricity to remove the dust from the panel by applying energy only to the particular matter being collected and therefore is very efficient in its applications unlike conventional cleaning. This increases the solar panels insolation absorption ability. The weight sensor continuously monitor the weight of the dust on the panel and Arduino controller gives the command to clean the dust when defined feedback is received from the sensors. Electrostatic precipitators work by ionizing the dust on the surface area of solar panel and forcing dirt particles towards the electrodes which are like electrical terminals. The first electrode is charged to a very high negative voltage and other to the positive. The positively charged electrode collects the dust particles. The particles attached to the positively charged plates are expelled or removed periodically to keep the panel dust free either manually or automatically.
\end{abstract}

Keywords: Energy scenario, Dust cleaning, Solar panel performance, Electro static precipitator 


\section{Introduction}

The use of solar PV panels is increasing in the present days due to the ever increasing energy demands, cost of energy and non availability of continuous power supply in the energy system. Now a days wide variety of appliances based on solar PV is increasing in the market at moderate prices namely, solar lanterns, solar heaters, blowers, coolers or fans, networking instruments, net metering etc. The trend in using the solarl PV is still expected increase in the near future as the research and choice of materials is going on and could lead to solar power plants to generate sufficient electricity for the local and regional needs. Considering the structure of the solar PV panel, it consists of transparent glass pane on its top which traps the solar radiations and reflects the radiations into the panel but if glass transparency gets affected by physical means then the absorption rate reduces and leads to reduced conversion efficiency [1], [2]. Cleaning of solar panels after the installation on the roof of the house, industry, shops is very difficult as dust particles do not allow the solar radiations to enter in the panel properly resulting reduced conversion efficiency of the panel leading to increased charging duration of batteries. Because of the above said effect there is a need for the proper maintenance and cleaning of solar panels to keep working efficiently. The cleaning of solar panel by using conventional methods like, vacuum cleaning or manual wipe/cleaning is not easy and in some cases not feasible because of typical mounting and mechanisms. The accumulated dust forms a layer on the panel due to which the solar radiations cannot reach properly into the PV panel receiver even though sufficient exposure to the sun is made resulting an inefficient conversion. In this regard, electrostatic precipitator (ESP) consisting of fixed electrodes can be used to remove the dust particles accumulated. The ESP is placed on the sides of the panel and the PV panel is mounted with weight sensor which regularly senses the dust thickness in terms of change in weight of the panel. If the weight of dust goes more than the predefined value, then the Arduino micro controller signal the ESP to start separating the dust and collect at positively charged electrode for the final disposal. In the ESP, the first electrode is charged to a very high negative voltage as the dirt particles move to the vicinity acquire negative charge and the second electrode charged to high positive voltage to attract the negatively charged dust particles. The dust particles which are accumulated at the positively charged electrode are further disposed by vibrating mechanism or a necessary brushing mechanism or cleaned manually. The common practices are,Vacuum suction cleaning, Manual wipe or cleaning which are difficult to practice and some time inefficient. Arduino based electrostatic precipitation method cleans the panel at periodic intervals intelligently whenever the panel is covered with the dust and exceed the predifined level [3], [4]. EPS is mainly used in those dusty atmosphere like cement industry, ceramic industry and fertilizer or powder industries etc where human operator can not perform cleaning due to various reasons. The EPS is used to collect the dust particles and the smoke particles and then the gases fumes are liberated to the atmosphere. This concept of absorbing the dust particles can be applied to remove the dust particles which are accumulated upon the solar panel. As the precipitator electrodes forms a field around it, the dust particles get charged and it get collected at postive electrode [4], [5].

\section{Comparative Study of Different Cleaning Methods}

Solar Photovoltaic panels are constantly exposed to various types of weather conditions throughout the year and are thus the target for dirt, dust, industrial residues, atmospheric pollution, algae, mosses, bird droppings etc. The agents and chemicals used during cleaning of the surfaces attract dirt and accelerate the deterioration process with negative repercussions on the appearance and mainly on the function of the PV panel as shown in Figure 1. The presence of these elements on the panel's surface prevents the sun's rays from filtering onto the panel's photovoltaic cells completely, reducing the solar performance and therefore efficiency.

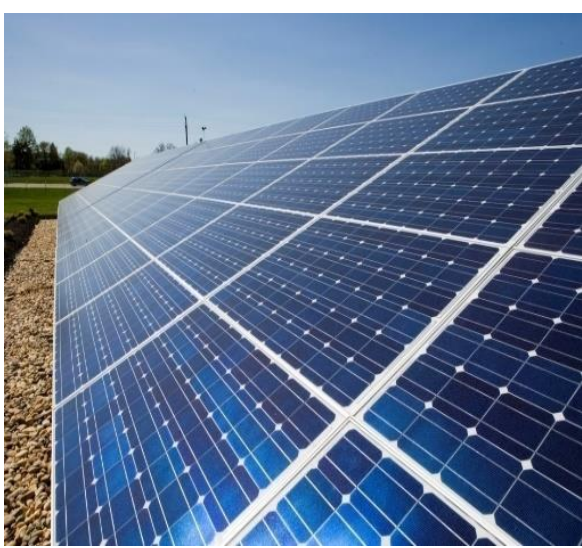

Figure1: Solar PV panel 


\subsection{Manual Cleaning}

This method require human operator to clean manually with the help of mopp or any wipers with suitable support structures as shown in Figure 2. The quality of cleaned surface is judged by visual method by the operator himself for the satisactory level or till the dust particles get wiped out completely.The process is found to be very tedious and challeging as the solar power plants consists of numbers of panels installed at a height of 12 to 20 feet or more from the ground. The time required and safety of the person and panel is in threat. To clean the panels manualy the fluids like cleansers or gels has to be used which act upon the panel and reduces the surface transparency if cleaning is not proper. There are quite chances of physical damages to the PV panels which cannot be avoided.

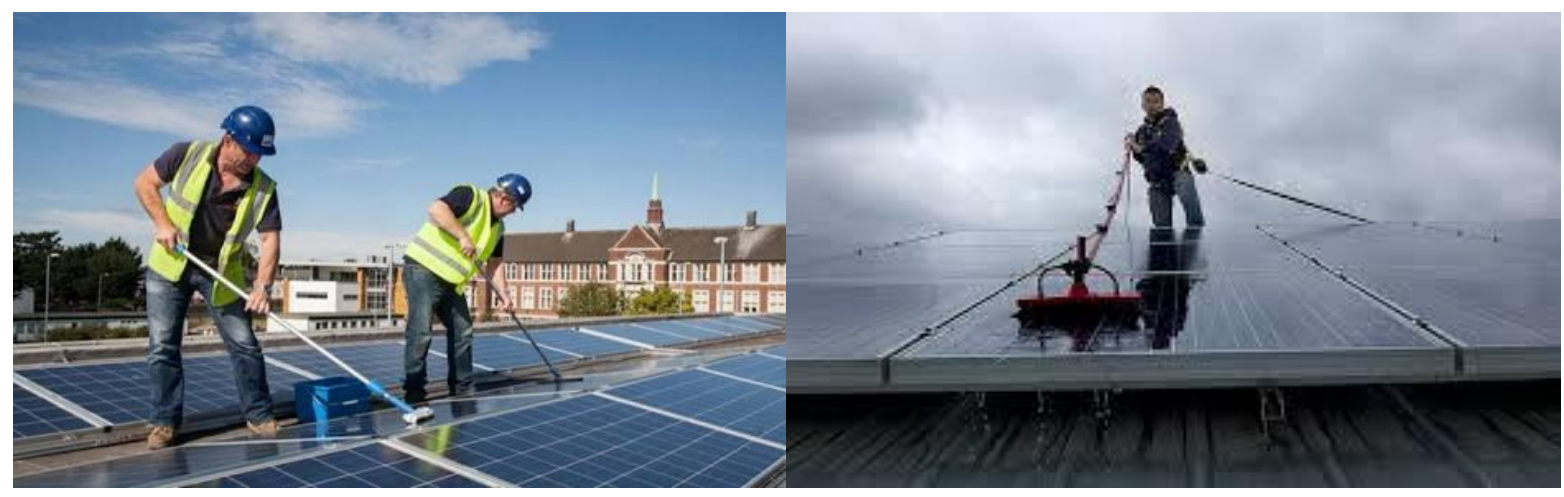

Figure 2: Manual cleaning

Figure 3: Cleaning using vaccum suction

\subsection{Vacuum Suction Cleaning}

A vacuum suction cleaner is a device that uses an air pump to create a partial vacuum to suck up dust and dirt, usually from floors, window panes etc.In general the electrical supply is given to the vacuum cleaner motor which creates the suction pressure. The power consumption of the vacuum cleaner is in watts and it doesnot justify the effectiveness of the cleaner. The input power is converted into airflow at the end and is measured in air watts. The vacuum cleaner can clean the panel properly only on the surfaces other than the corners and this has to be handled manually as shown in Figure 3. The proper training to the operator is necessary as the physical movements on the panel with cleaner is inevitable. Over a period of time scratches and accumulated dust cause inefficient absorption of solar insolation.

\subsection{Automatic Wiper Based Cleaning}

The automatic wiper based cleaning incorporate a rubber wiper and water pot for the spray of water with additives and cleaning. The process is exactly like vehicle glass cleaning and require a automatic mechanism to operate and complete the task. Mechanism is battery operated as shown in Figure 4. This method is simillar to earlier one and operated automatically by the suitable control mechanism but the impacts are simillar to those earlier ones.

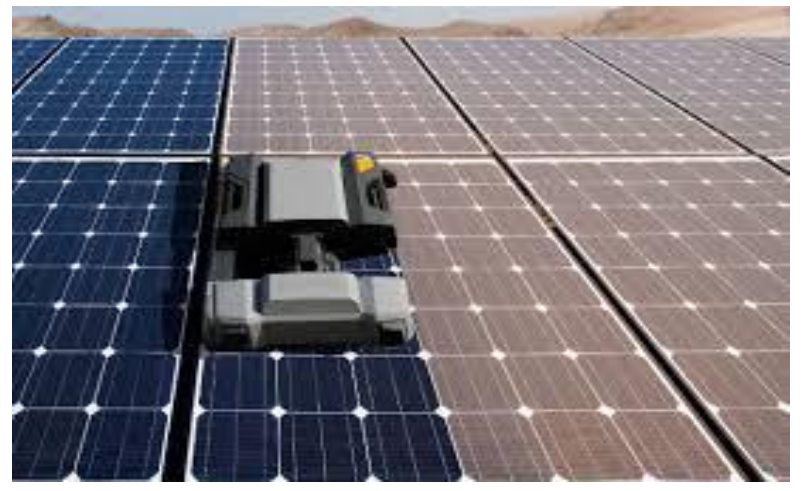

Figure 4: Automatic cleaner

\section{Electrostatic Precipitator}

Considering the impacts of different cleaning methods for solar PV panels the mechanical damages, scratches and scars like marks the electrostatic precipitator work with non-contact mechanism. It cleans efficiently and protects the top surface of the panel without any physical contact. An electrostatic precipitator (ESP) is a filtration device that removes fine dust particles from the surface of the solar PV panel using the force of an induced electrostatic charge. The mechanism consists of the electrodes which 
Hudedmani et al., Adv.J. Grad. Res.; Vol. 1 Issue 1, pp: 24-29, January 2017

are charged suitably as shown in Figure 5. The electrodes of the ESP obtain power through relay when the Arduino controller signals after obtaining weight of the PV panel after the comparison with preset value. The negative electrodes of the electrostatic precipitator induces the negative charge on the dust particles which are present on the surface of the panel. The dust particles are accumulated at the positive electrode after being attracted. In this way, a non-contact dust cleaning technique helps in improving the efficiency of solar panel.

\subsection{Working Principle of ESP}

The plate precipitator is one of the types of electrostatic precipitators because of its compact size and its suitability to place it on the PV panel. The ESP consists of electrodes which are connected to the voltage source as shown in Figure 5. The power supply and converters for obtaining higher voltage are not shown and discussed in this article. The electrodes are fixed in nature and create field in the vicinity to ionize the dust particles.

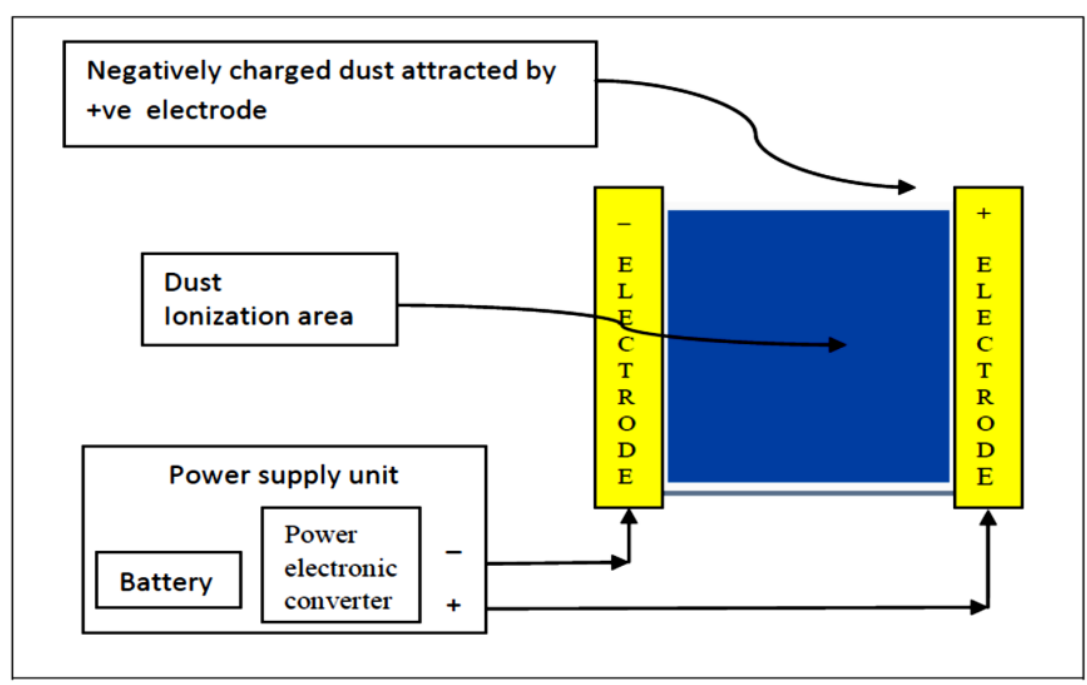

Figure 5: ESP block diagram

The negative electrode charges the dust particles with the negative charge on the surface of the solar panel. Due to the attraction of unlike charges dust particles are collected at positive electrodes. This principle of ESP is used in the present work where the electrodes are placed on the opposite sides of the panel as shown in Figure 5. Whenever the dust particles accumulate on the panel and based on the weight of the dust on the panel which is sensed by the weight sensor fitted. The Arduino controller enables the signal to ESP supply to be applied to the electrodes for the collection of dust. The predetermined level of weight signal is always verified by the controller as and when it receives the signal.

\subsection{Electrostatic Precipitator Based Solar PV Panel Cleaning}

The ESP concept is configured with Arduino micro controller with necessary hardware. Figure 6 represents the overall block diagram and Figure 7 represents the Arduino interface with weight sensor.

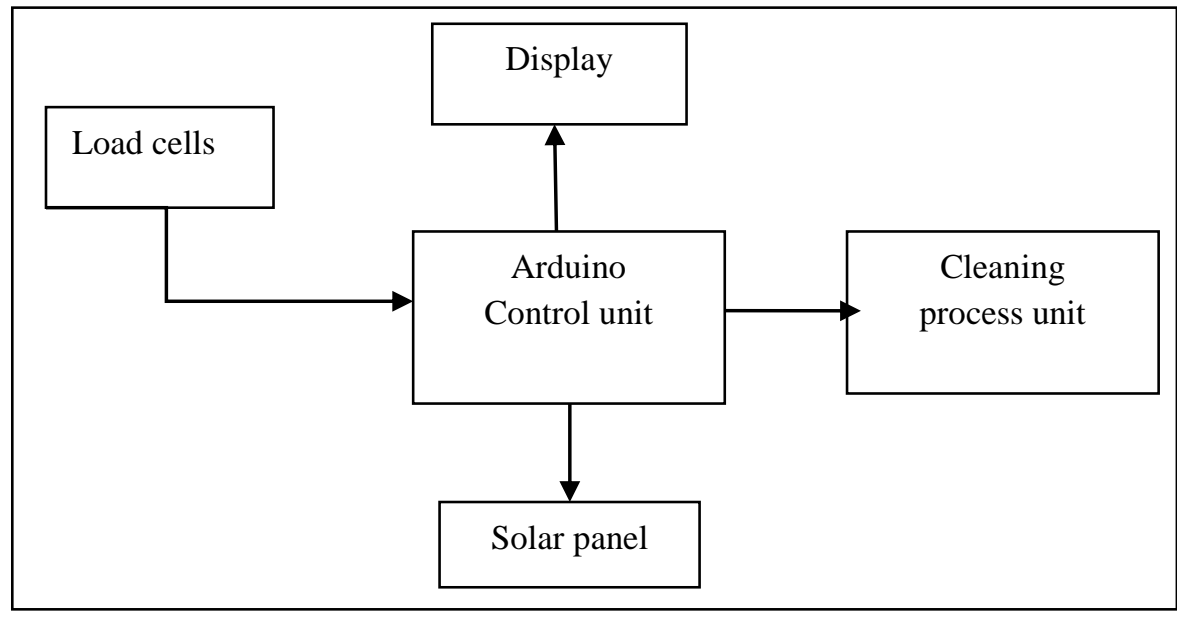

Figure 6: Block diagram of Arduino based ESP 


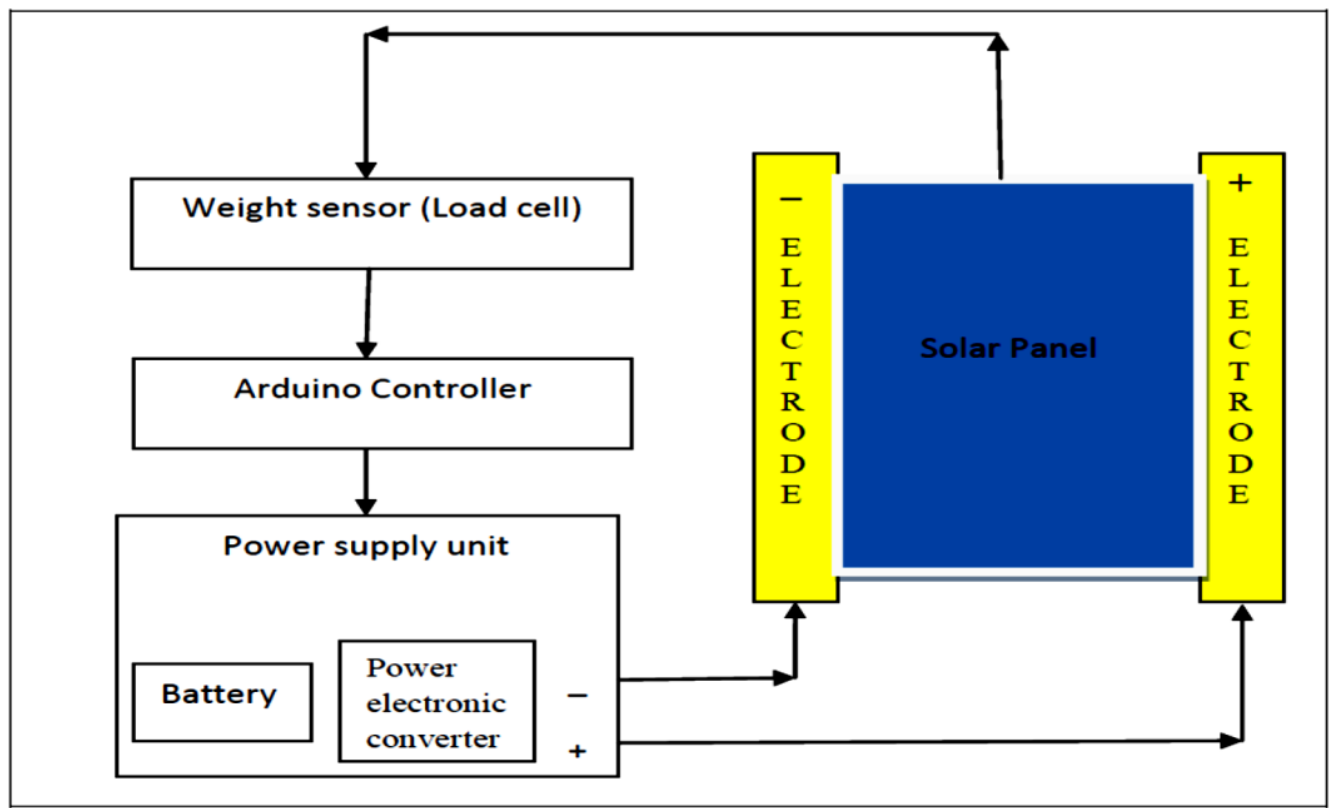

Figure 7: Arduino interface to ESP

\subsubsection{Arduino Microcontroller}

It is the intelligent controller used in the ESP. When the weight of panel increases due to accumulation of dust the weight sensor activates the Arduino as shown in Figure 7. The Arduino energizes the electrodes of precipitator by applying power input to the electrodes to ionize the dust particles and attract them. Later with suitable method collected dust particles are disposed from the electrodes. The picture of the Arduino controller is shown in Figure 8.

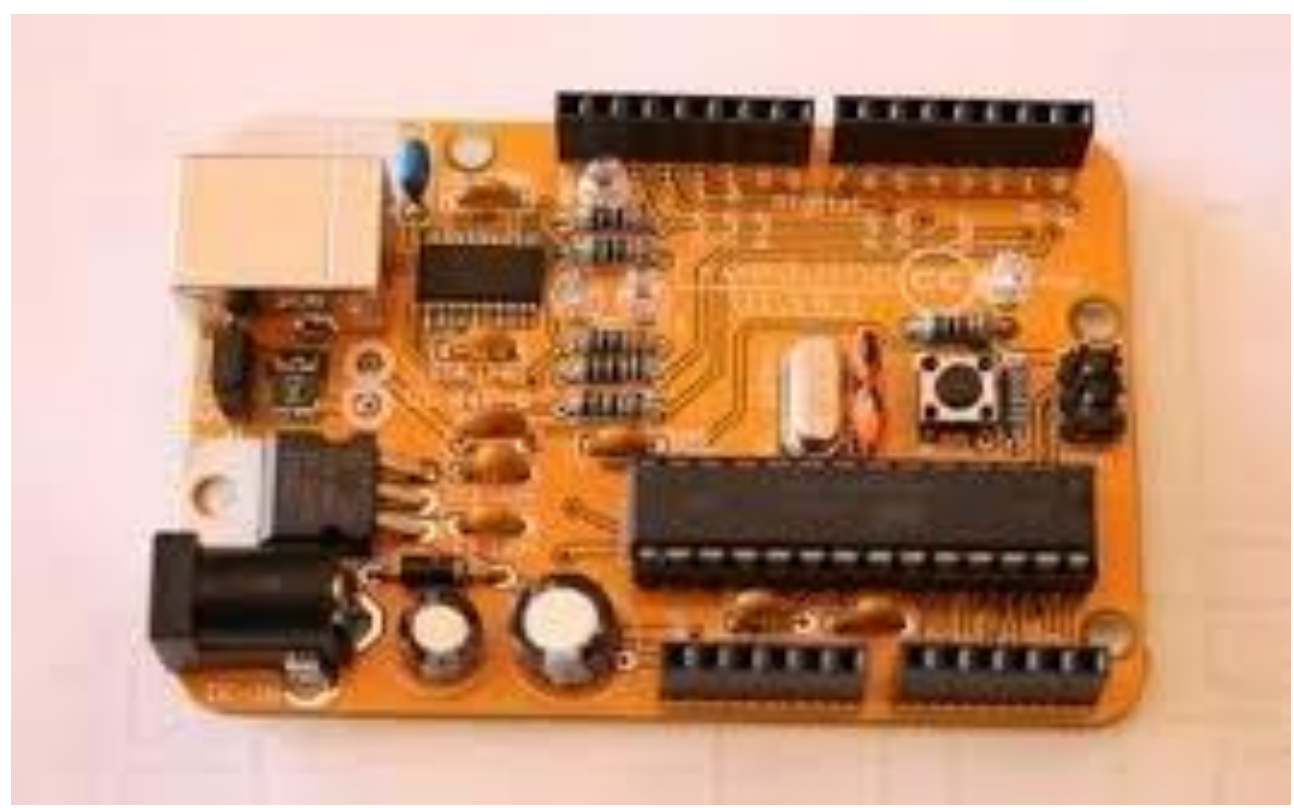

Figure 8: Arduino UNO kit

\subsubsection{Weight Sensor}

The tare weight of solar PV panel is input to the Arduino controller for its reference. The accumulated dust on the solar panel increases the panel weight. The continuous monitoring of the weight of the solar panel by the Arduino controller using load cell enables the working program for ESP to collect the dust particles at the electrodes. The load cell is mounted below the panel and supported by mounting plate. 


\section{Conclusions}

As the scarcity of energy is increasing and decline of energy resource result an opportunity to incorporate new and renewable energy systems in practice. In this regard use of solar PV system is becoming promising as availability of PV solar based devices is increasing in the market. Considering the dust accumulation problem of solar PV panel a comparative study of different cleaning techniques is done. Electro static precipitator with Arduino controller enhances the efficiency of solar panel and thus helps in utilizing the solar energy effectively and efficiently. Further, as a scope of improvement; a movable electrodes mechanism can be worked out in which electrode roll over the solar panel like a computer scanner head movement to collect and clean the dust particles to the greater extent.

\section{How to cite this article:}

Hudedmani, M., Joshi, G., M, U., \& Revankar, A. (2017). A Comparative Study of Dust Cleaning Methods for the Solar PV Panels. Advanced Journal of Graduate Research, 1(1), 24-29. doi: https://doi.org/10.21467/ajgr.1.1.24-29

\section{References}

[1] S. A. Sulaiman, H. H. Hussain, N. S. H. Nik Leh, and M. S. I. Razali, "Effects of Dust on the Performance of PV Panels," Int. J. Mech. Aerospace, Ind. Mechatron. Manuf. Eng., vol. 5, no. 10, pp. 2028-2033, 2011.

[2] D. Singh Rajput and K. Sudhakar, "Effect Of Dust On The Performance Of Solar PV Panel," Int. J. ChemTech Res., vol. 5, no. 2, pp. 1083-1086, 2013.

[3] G. Librandi, J. Narain, and H. Yu, “Autonomous Photovoltaic Panels Cleaning System,” Brooklyn, NY, 2012.

[4] A. Mizuno, "Electrostatic precipitation," IEEE Trans. Dielectr. Electr. Insul., vol. 7, no. 5, pp. 615-624, 2000.

[5] N. Grady B. and O. J. Sabert M, "Method of charging an electrostatic precipitator," US5972076 A, 1999.

\section{Publish your research article in AIJR journals-}

$\checkmark \quad$ Online Submission and Tracking

$\checkmark$ Peer Reviewed

$\checkmark$ Rapid decision

$\checkmark$ Immediate Publication after acceptance

$\checkmark$ Open Access (Articles freely available online)

$\checkmark$ Retain full copyright of your article.

Submit your article at journals.aijr.in 